\title{
PROGRESS WITH SNS FAST BEAM CHOPPER*
}

\author{
S.S. Kurennoy, J.F. Power, R.J. Roybal, and D.L. Schrage, LANL, Los Alamos, NM 87545, USA
}

\begin{abstract}
The fast traveling-wave $2.5-\mathrm{MeV}$ beam chopper for the Spallation Neutron Source (SNS) project has a new current structure based on the meander-folded notched stripline with separators. Measurements of the full-length structure prototype showed a good agreement with predictions of MAFIA electromagnetic modeling [1]. The design of the final chopper units is completed, and they are being manufactured. Here we report on the final design of the chopper and on results of recent thermal and vacuum tests of the prototype chopper structure.
\end{abstract}

\section{INTRODUCTION}

The SNS is a next-generation pulsed spallation neutron source designed to deliver up to $2 \mathrm{MW}$ of beam power on the target at $60 \mathrm{~Hz}$ [2]. Its design stipulates a 1-GeV linear $\mathrm{H}^{-}$accelerator and an accumulator ring. The SNS storage ring accumulates the linac beam during a few hundred turns (a macropulse, $1 \mathrm{~ms}$ ) using $\mathrm{H}^{-}$injection through a carbon foil. The beam injected into the ring is stacked into a single long bunch. The linac macropulse must be chopped at the ring revolution frequency, around $1 \mathrm{MHz}$, to provide a gap required for the ring kicker rise time during a single-turn ring extraction. The final clean beam chopping in the linac is to be done by a fast chopper in the Medium Energy Beam Transport (MEBT) line.

The MEBT is designed to transport up to $52 \mathrm{~mA}$ of peak beam current from a $2.5-\mathrm{MeV} 402.5-\mathrm{MHz}$ RFQ to a drift-tube linac. The traveling-wave MEBT chopper fills the space between its two mirror-symmetric current structures, one carrying a positive and one negative voltage pulse, with a wave of the deflecting electric field that propagates along the beam path at the same speed as the beam does, $v=0.073 c$. This is achieved by sending the wave along the meander-folded transmission line. The initial requirements [2] called for a 50-cm long MEBT chopper with the rise and fall time below $2.5 \mathrm{~ns}$ to avoid partially-chopped bunches. MAFIA simulations [3,1] have shown that the notched meander-line structure can provide the rise and fall time in the 1-ns range. It was confirmed by the measurements of the $50-\mathrm{cm}$ prototype meander-line current structure [1]. The main difficulty to achieve a short chopper rise time, however, is not with the structure itself, but with the pulse generator.

Later beam dynamics simulations have shown that partially chopped bunches would not lead to extra beam loss in the linac or the ring transfer line. In the most recent MEBT design the chopper length was reduced to $35 \mathrm{~cm}$, while the inter-plate gap was increased from 15 to $18 \mathrm{~mm}$.

\footnotetext{
*Work supported by Office of Basic Energy Science, the US Department of Energy, and by ORNL.
}

A shorter chopper improves the MEBT beam dynamics. To provide the designed beam deflection of $18 \mathrm{mrad}$, the requirement for the pulser voltage was changed from 1.5 to $2.35 \mathrm{kV}$ with a slower rise and fall time, below $10 \mathrm{~ns}$, thus allowing up to 3 partially-chopped bunches in the beginning and the end of each chopper pulse, which lasts around $300 \mathrm{~ns}$.

\section{MEANDER CURRENT STRUCTURE}

The current structure based on the meander-folded notched stripline with separators was described in detail in Refs. [3,1]. It is illustrated in Fig. 1. The notched meander line provides the proper wave phase velocity ( $\beta=0.073$ ) along the beam path while keeping the characteristic impedance of the line equal to $50 \Omega$.

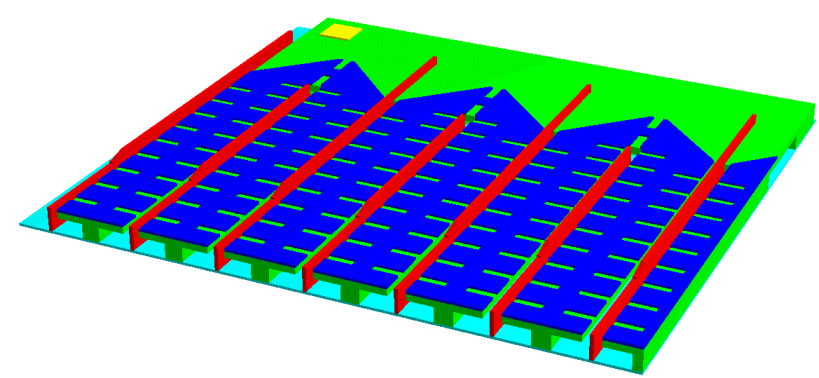

Figure 1: A part of the meander structure model: notched metal meander strip (dark-blue) on dielectric supports

(green), metal separators (red) are connected to the ground plate (light-blue, below).

A photograph of the 50-cm prototype of the meanderline current structure is presented in Fig. 2.

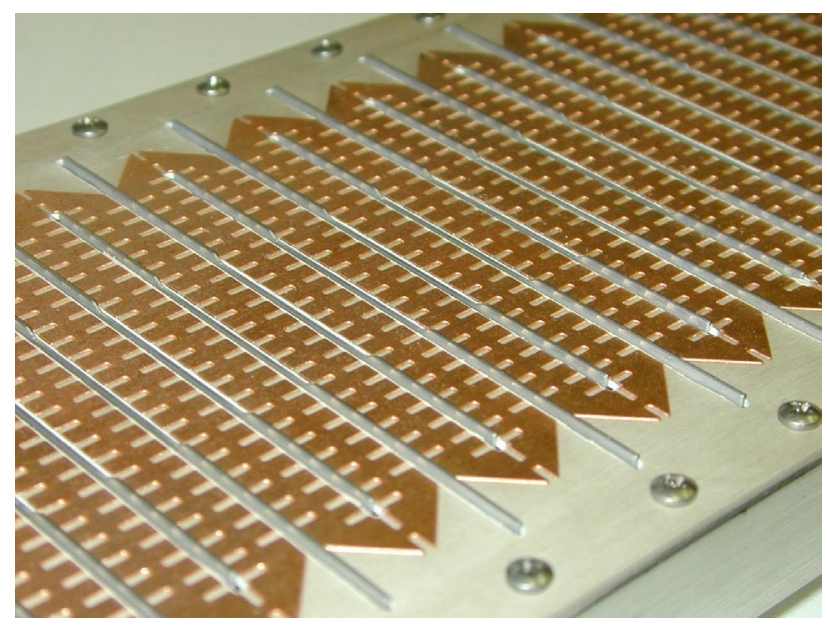

Figure 2: Close-up view of the prototype current structure. 
The notched meander line is supported by a T-shaped dielectric support that goes all along the stripline length. The support is carved from a continuous dielectric plate of Rogers' duroid RT/6002, after the notched meander pattern is chemically etched on the copper coating of the dielectric plate. The copper thickness in the transmission line is $0.25 \mathrm{~mm}$, and the dielectric is $2.5 \mathrm{~mm}$ thick. The metal width in the line is $8 \mathrm{~mm}$, and the meander period is $1 \mathrm{~cm}$; it leaves $2-\mathrm{mm}$ gaps between the straight strips. The grounded metal separators protrude into these gaps through the narrow cuts in the dielectric to reduce the coupling between adjacent pieces of the meander line. The notches on the line serve to slow down the TEM wave along the line straight sections to $0.68 c$. The notches are 3-mm deep and 1-mm wide, and their period is $4 \mathrm{~mm}$. The wave phase velocity along the beam can be adjusted to $0.073 c$ by choosing the meander width in the direction transverse to the beam. This width was $98 \mathrm{~mm}$ in the 50 $\mathrm{cm}$ prototype, and after the measurements [1] it is adjusted to $96 \mathrm{~mm}$ for the final units. The whole structure is clamped by bolts near its sides to the metal ground plate, and the dielectric supports are glued to the ground plate with a special epoxy to provide the required line flatness. The ground plates will be fabricated of 6061-T6 aluminum alloy.

\section{CHOPPER FINAL DESIGN}

The chopper pulser has now the peak voltage $2.35 \mathrm{kV}$, higher by factor 2.6 compared to its original specification of $900 \mathrm{~V} \mathrm{[2],} \mathrm{so} \mathrm{that} \mathrm{the} \mathrm{peak} \mathrm{current} \mathrm{through} \mathrm{the} \mathrm{meander}$ line is about $47 \mathrm{~A}$. As a result, the power dissipation in the chopper structures requires an extra attention. Analytical estimates of the dissipated power in the chopper with the account of the current pulse frequency spectrum give values between 8 and $12 \mathrm{~W}$ per current plate, depending on a model of the line losses as a function of frequency. The measurements of S-parameters in the $50-\mathrm{cm}$ prototype structure with a network analyzer, scaled down to the length of $35 \mathrm{~cm}$, give a higher estimate of the power loss, about $19 \mathrm{~W}$. In any case, this power cannot be dissipated by radiative cooling without the temperature increase of the current plates by more than $100^{\circ} \mathrm{K}$, which would be unacceptable for the epoxy bonding material. Therefore, an active water cooling was proposed. A thermal and structural analysis of the chopper current structures has been performed [4]. It was demonstrated that a simple cooling scheme, with one 0.25 " channel down the short direction in the ground plate and the water flow rate of 0.5 GPM, is sufficient to keep the maximal temperature increase anywhere on the current plate below $1.5^{\circ} \mathrm{K}$. Related stresses and displacements are very small.

The final design of the chopper is shown in Fig. 3. The gap between the current structures is fixed by inserted spacers. One can see the water pipes, thermocouples, and protective scrapers on both ends of the chopper. This assembly will be mounted on a vacuum-box lid as shown in Fig. 4 . The chopper vertical position can be slightly ad- justed with a linear feedthrough. The lid with the current structures will be assembled at LANL and then shipped to LBNL for installation in a vacuum box in the MEBT.

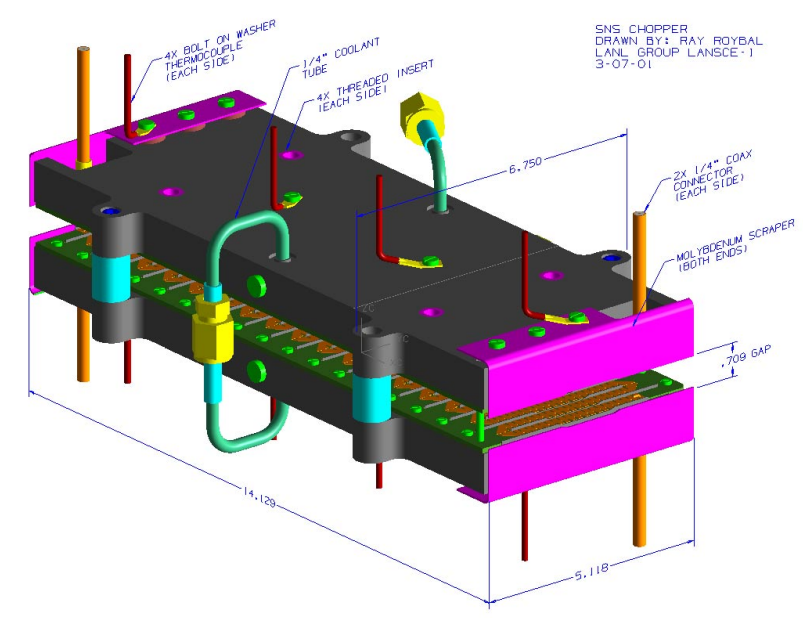

Figure 3: Assembled current plates of the MEBT chopper.

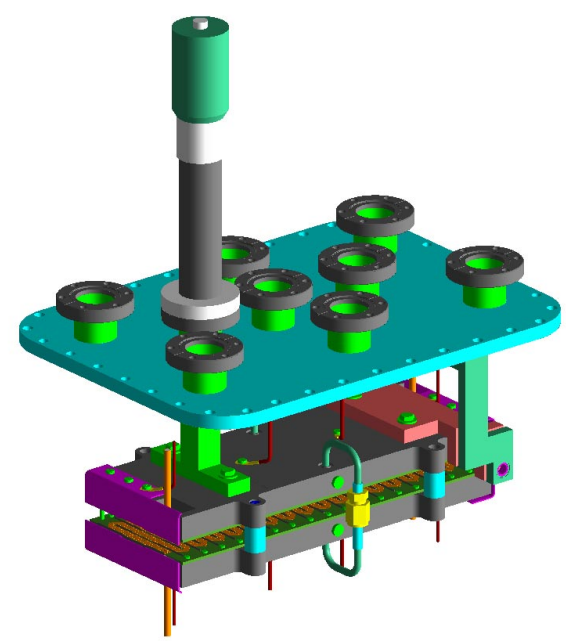

Figure 4: Chopper assembly mounted on the vacuum box lid (courtesy of Daryl Oshatz, LBNL).

The parts of the two units, chopper and antichopper, the meander lines on the carved dielectric supports and the ground plates - have been manufactured. They are now in the process of inspection before the final assembly.

\section{VACUUM TEST}

In the meantime, an important vacuum test has been performed on the $50-\mathrm{cm}$ prototype current structure. The reason for this test was a concern that the dielectric support material, duroid RT/6002, and the epoxy, could be not very good from the vacuum standpoint.

The chopper current structure assembly, after cleaning outer surfaces from fingerprints and residues by wiping them down with acetone and alcohol, was placed in a vacuum test chamber, see Fig. 5. Then the chamber was pumped under vacuum for three weeks. After 24 hours the system pressure was $6 \cdot 10^{-7}$ Torr. The steady state at $3 \cdot 10^{-7}$ Torr was reached after 5 days. The steady-state outgassing 
rate for the current structure assembly was calculated to be about $2 \cdot 10^{-5}$ Torr $\cdot$ liter/sec at room temperature [5], which is a rather low value given that there were no special cleaning procedures in the process of manufacturing the structure prototype. One can expect even lower outgassing rates for the production units, assuming rigorous cleaning during various fabrications steps.

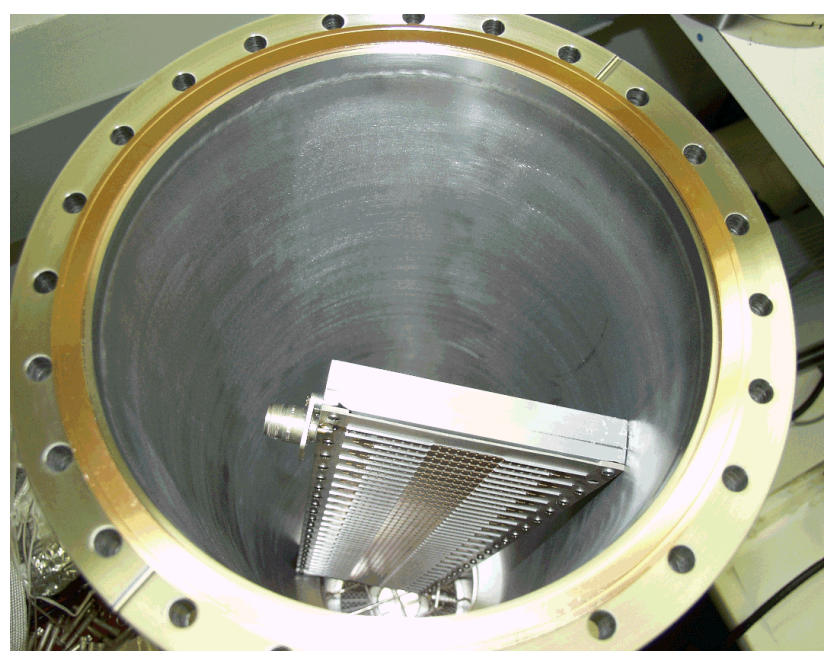

Figure 5: The prototype current structure in the vacuum test chamber.

\section{PULSE GENERATORS}

The voltage pulse generators with the maximum voltage $3 \mathrm{kV}$ and the rise and fall time below $10 \mathrm{~ns}$ have been ordered from Directed Energy, Inc (Fort Collins, CO) with expected delivery in April 2001. Unfortunately, the order was delayed, and the new delivery date is late July 2001.

Parts of the pulse generating system have been purchased and tested, including the $50-\Omega$ cables, connectors, and pulser loads. We plan to use Bird 8775 water-cooled loads rated for $50 \mathrm{~kW}$, see Fig. 6 . The high-power rating is required to handle $180-\mathrm{kW}$ peak power available from our pulse generators. These loads have a peak voltage rating of at least $3.5 \mathrm{kV}$ for $1-\mu \mathrm{s}$ pulses, exceeding our requirement. We have tested two of these loads at low voltages, measuring their frequency response with a network analyzer and their transient response with a time-domain reflectometer (TDR). The measured impedance of the loads is within the specifications, $50 \pm 0.5 \Omega$.

The equipment rack location will require approximately $12 \mathrm{~m}$ of cable between the pulse generator outputs and the chopper structure inputs. Andrew's 1-5/8" diameter HJ750A Heliax cable has been selected for its low loss at the frequencies of interest. For 10-ns rise times, the highest frequency of interest is on the order of $35 \mathrm{MHz}$. The highest frequency of interest in regards to settling time is about half of that, or $17.5 \mathrm{MHz}$. We need the pulses to settle to within something like $0.5 \%$, which implies a maximum loss of $0.044 \mathrm{~dB}$ for the cable to have a negli- gible effect on the settling time. We are using 7-16 DIN connectors on the output cables and loads.

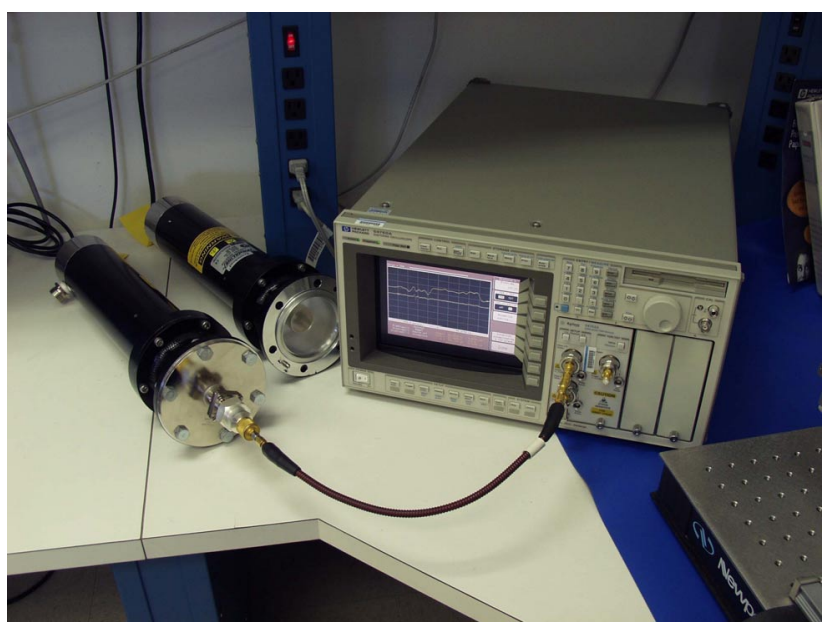

Figure 6: Bird Electronics 8775 load is being tested with the time-domain reflectometer.

\section{CONCLUSIONS}

The progress with development of the fast travelingwave $2.5-\mathrm{MeV}$ SNS beam chopper with the new current structure (the meander-folded notched stripline with separators) is reported. Thermal and vacuum tests of the 50$\mathrm{cm}$ prototype chopper structure have been performed. It was decided to use active water cooling of the chopper current structures. The vacuum tests of the prototype showed a rather low outgassing rate. Based on results of the prototype tests, the design of the MEBT chopper and antichopper is finalized. Their fabrication process is also close to completion.

The authors would like to thank R. LaFave and S. Hopkins for their contributions to the chopper development.

\section{REFERENCES}

[1] S.S. Kurennoy and J.F. Power. "Development of Meander-Line Current Structure for SNS Fast 2.5-Mev Beam Chopper," Proceed. EPAC'00, Vienna, 2000, p. 336.

[2] NSNS Collaboration, "NSNS Conceptual Design Report", NSNS-CDR-2/V1, Oak Ridge, TN (1997); see also at URL http://www.sns.gov

[3] S.S. Kurennoy, J.F. Power, and D.L. Schrage, "Meander-line Current Structure for SNS Fast Beam Chopper," Proceed. PAC' 99, New York, 1999, p.1399.

[4] R. LaFave, "Structural / Thermal Analysis of SNS Chopper," LANSCE-1:00-91, Los Alamos, 2000.

[5] S. Hopkins, Private communication, April 2001. 